\title{
FULLY BATCH-FABRICATED LINEAR QUADRUPOLE MASS FILTERS
}

\author{
K. Cheung, L. F. Velásquez-García, and A. I. Akinwande
}

Massachusetts Institute of Technology, Cambridge, MA 02139 USA

\begin{abstract}
We report the design, fabrication, and characterization of a fully batch-fabricated linear quadrupole mass filter. The device demonstrates for the first time, a microfabricated quadrupole mass filter that doesn't require any post-fabrication electrode assembly. Utilizing a design with non-conventional square electrodes, a 30 $\mathrm{mm}$ quadrupole with an effective electrode diameter of $\sim 1.7 \mathrm{~mm}$, and integrated ion optics was fabricated. At a driving frequency of $2 \mathrm{MHz}$ and a maximum applied voltage of $100 \mathrm{~V}$, a mass range of 250 a.m.u. was achieved in the first stability region, and a halfheight peak-width of 0.7 a.m.u. was obtained in the second stability region at mass 28 .
\end{abstract}

\section{INTRODUCTION}

Mass spectrometers are powerful analytical tools that can identify the composition of unknown compounds, and quantify compounds that are known. Scaling-down this device will permit faster analysis times, power savings through operation at higher pressures, portability, and reduced costs $[1,2]$. A popular method of miniaturization is through the use of microelectromechanical systems (MEMS) fabrication technologies because it permits batch-processing. The development of micro-scaled gas analyzers will have applications in national security, environmental safety and monitoring, satellites and space exploration, industrial processing, as well as biological research and healthcare.

Mass spectrometers comprise primarily of an ionizer to create charged species, a mass filter to sort the ions according to their mass-to-charge ratios, and a detector to measure the quantity of the filtered ions. Batch-fabricated ionizers and detectors have already been developed for a micro-gas analyzer system $[3,4]$. In order to produce these systems inexpensively and efficiently, the mass filter component should also be completely batch-fabricated.

Linear quadrupoles are popular mass filters due to their simplicity in design and usage. Researchers have demonstrated mass filtering with MEMS-based quadrupole mass filters (QMFs) [5-7]. Reported designs utilize metallic rods or metalized glass rods as electrodes, which required manual assembly of these electrodes after the MEMS components were fabricated. This methodology places a bottleneck on the manufacturing and sets a limit on the precision that can be achieved.

We propose using a non-conventional square electrode geometry that can be fully batch-fabricated, thus taking advantage of the dimensional precision associated with MEMS technology. This paper reports the design, fabrication, and characterization of the micro-square electrode quadrupole mass filter (MuSE-QMF). The operating principles of a QMF will be described, followed by an overview of the optimization process. The fabrication process flow will be presented, and the experimental results discussed.

\section{THEORY}

An ideal QMF utilizes four hyperbolic electrodes aligned in a symmetric manner to generate an ideal quadrupole field. If the applied potential is a combination of r.f. and d.c. voltages, the equations of motion for a charged ion in this field would be given by the Mathieu equation [8]. This equation has stable and unstable solutions that can be mapped as a function of two parameters. Overlapping the Mathieu stability diagrams for the directions orthogonal to the quadrupole axis define stability regions, shaded areas in Fig. 1, where ion motion is stable in both directions.

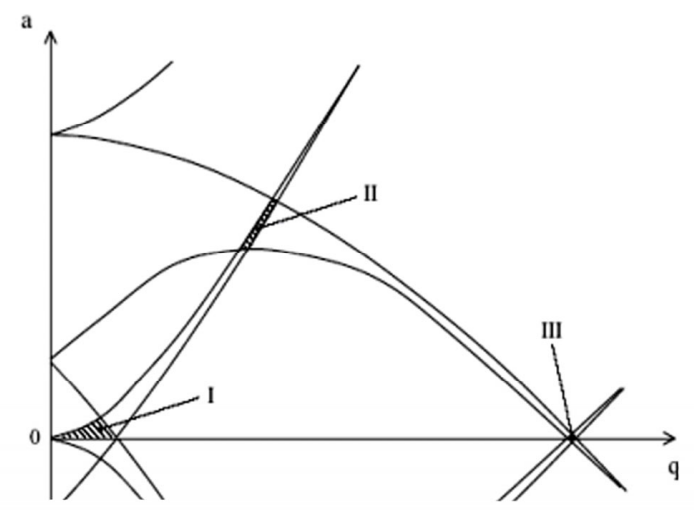

Figure 1: The Mathieu stability diagram showing quadrupole stability regions I, II, and III. The Mathieu parameters, $a$ and $q$, are functions of the device geometry and the operating conditions.

(a)
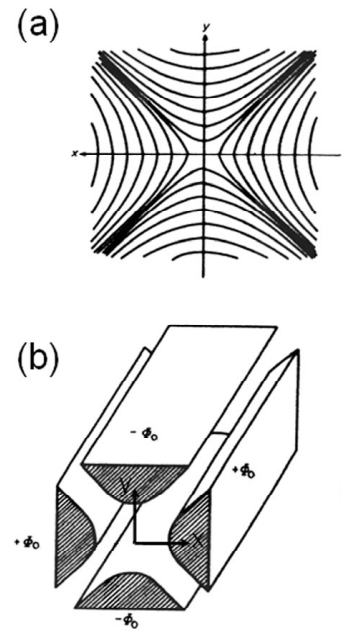

$\phi_{0}=\mathrm{U}+\mathrm{V} \cos (\omega \mathrm{t})$

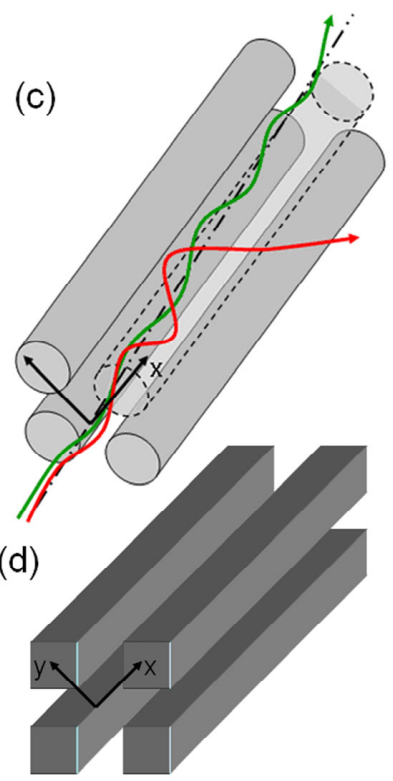

(c)

(d)

Figure 2: (a) Ideal quadrupole potential field [8], (b) Hyperbolic electrodes with standard applied voltages for an ideal QMF [8], (c) Conventional cylindrical electrode QMF (transmitted ion trajectories are in green), (d) Proposed square electrode geometry.

Most commercial QMFs and reported MEMS-based versions utilize cylindrical electrodes instead of hyperbolic ones due to the reduced complexity in manufacturing. To compensate for the distortion that comes from using non-hyperbolic electrodes, optimization was conducted to minimize the higher-order field components that are a result of this non-ideality. Continuing the technological progression shown in Fig. 2, optimization can be conducted on the MuSE-QMF to minimize unwanted field components as well.

QMFs are typically operated in stability region I but there are reports of higher resolution and improved peak-shapes that come with operation in stability region II $[8,9]$. Driving the MuSEQMF in a higher stability region can serve to further improve the performance of the device aside from the geometric optimization. 


\section{DESIGN AND OPTIMIZATION}

Analysis of the field components of the MuSE-QMF was conducted in a manner similar to that reported in [10]. A general schematic of the device was conceived and critical dimensions were parameterized as illustrated in Fig. 3. Maxwell 2D was used to calculate the potentials for the various geometries. The field data was then exported into a MATLAB script which decomposed the potentials into corresponding multipole terms.

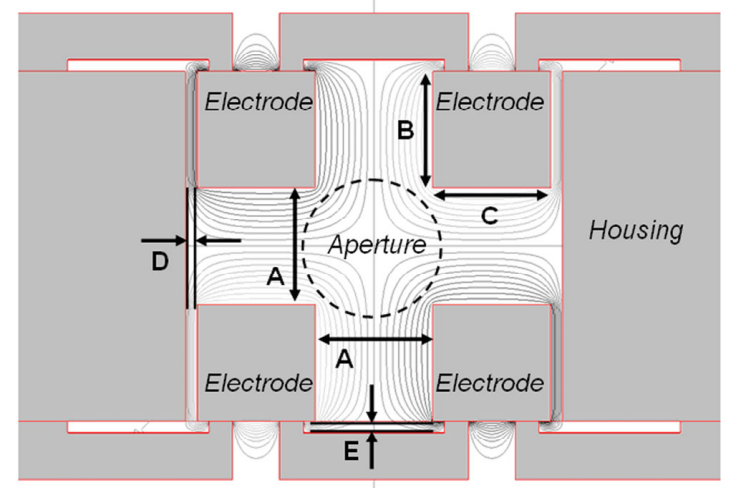

Figure 3: Cross section of the device geometry used during optimization, with field lines generated by Maxwell 2D. The critical dimensions are $A$ (the characteristic dimension of the quadrupole aperture), $B$ (the thickness of the electrodes), $C$ (the width of the electrode), $D$ (the horizontal electrode-to-housing spacing), and $E$ (the vertical electrode-to-housing spacing).

(a)

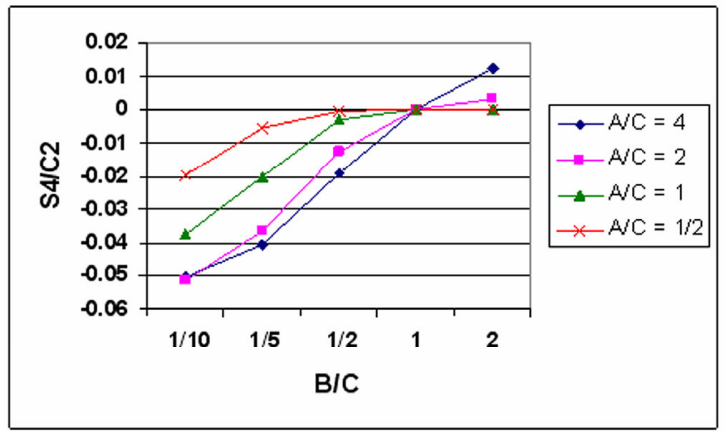

(b)

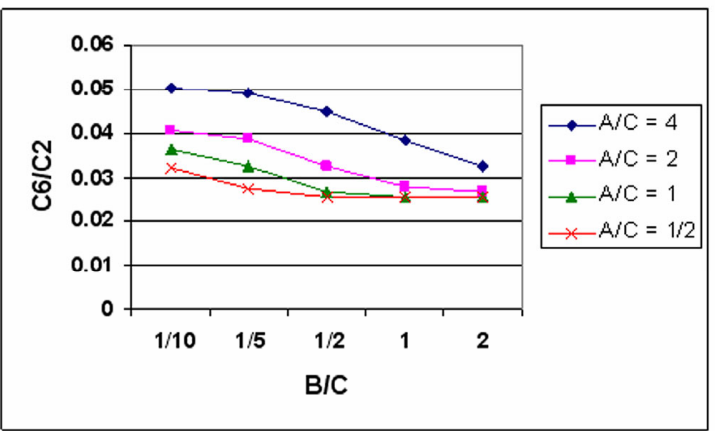

Figure 4: Optimization results using dimensions $A, B$, and $C$. Electrode dimensions and aperture size were parameterized without including the housing in the simulations.

From the boundary conditions established by symmetry of the electrodes, the first three significant terms in the expansion are $\mathrm{C}_{2}$, $\mathrm{S}_{4}$, and $\mathrm{C}_{6} . \mathrm{C}_{2}$ corresponds to the ideal quadrupole term, while $\mathrm{S}_{4}$ and $\mathrm{C}_{6}$ are the first odd and even higher-order terms respectively. The ratios $\mathrm{S}_{4} / \mathrm{C}_{2}$ and $\mathrm{C}_{6} / \mathrm{C}_{2}$ give an indication of the significance of the unwanted field components that should be minimized. The results shown in Fig. 4 led to the conclusion that setting the dimensions $\mathrm{A}=\mathrm{B}=\mathrm{C}$ will minimize the undesirable terms. Data in Fig. 5 show that $\mathrm{S}_{4} / \mathrm{C}_{2}$ is optimal when $\mathrm{D}=\mathrm{E}$, and $\mathrm{C}_{6} / \mathrm{C}_{2}$ is a minimum when $\mathrm{D}$ is large. For fabrication and testing considerations, dimension A was set to $1 \mathrm{~mm}$ and $\mathrm{E}$ to $100 \mu \mathrm{m}$. A large device aperture will increase the signal strength of the transmitted ions, while a small electrode-to-housing distance will improve processing uniformity.

Maximum transmission through a QMF occurs when the incoming ions enter near the central axis of the device [8]. The inclusion of integrated ion optics, as shown in Fig. 6, can help focus the ion stream towards the axis, as well as control the inlet and outlet conditions, thus improving overall performance.

(a)

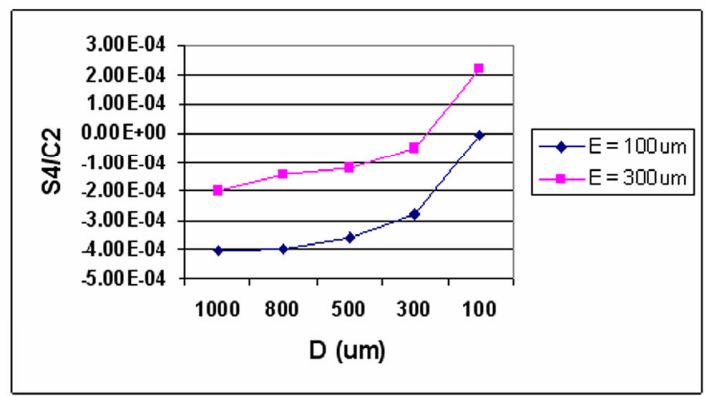

(b)

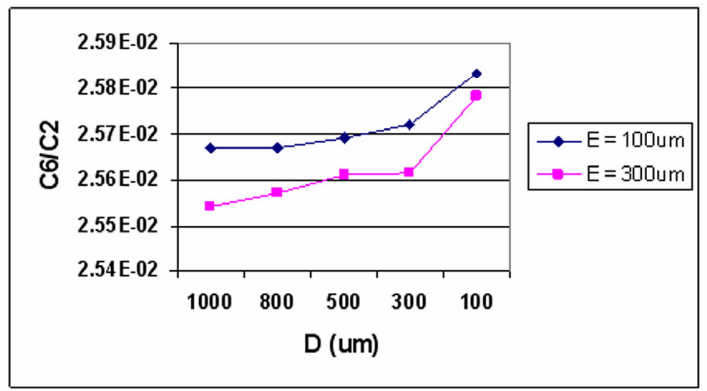

Figure 5: Optimization results using $A=B=C=1 \mathrm{~mm}$, while varying the dimensions $D$ and $E$. The full geometry shown in Fig. 4 is used in these simulations.

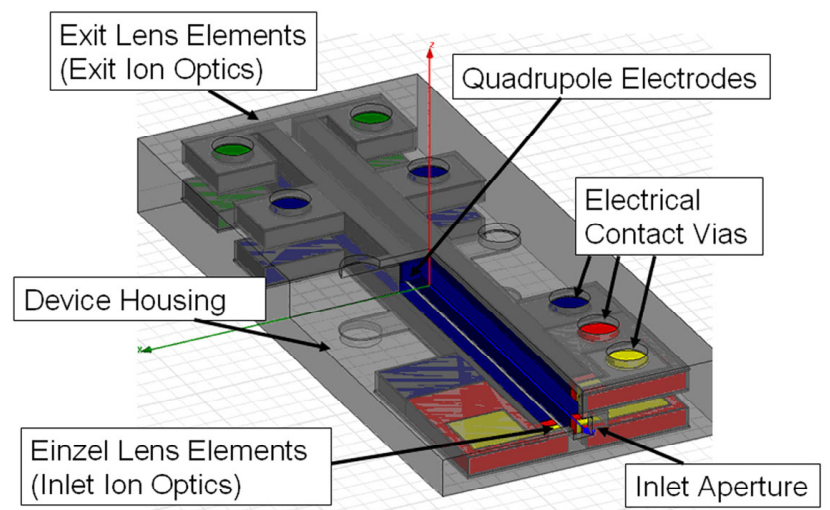

Figure 6: Schematic of the MuSE-QMF. The device has integrated ion optics denoted in red (Einzel lens 1 \& 3), yellow (Einzel lens 2), and green (exit lens). The electrodes are shown in blue.

\section{DEVICE FABRICATION}

Five highly-doped silicon double-side polished (DSP) wafers are needed to complete the device. Two $500 \pm 5 \mu \mathrm{m}$ wafers are 
used as the capping layers, two $1000 \pm 10 \mu \mathrm{m}$ wafers serve as the electrode layers, and another $1000 \pm 10 \mu \mathrm{m}$ is utilized as a spacer layer. All the wafers initially have $0.3 \mu \mathrm{m}$ of oxide to serve as a protective layer during processing.

A series of deep reactive ion etches (DRIE), wet thermal oxidation, and silicon fusion bonding is used to realize the device. The cap wafers are defined with release trenches $100 \mu \mathrm{m}$ deep that are required for the electrode etch (Fig. 7a), and through-wafer vias for electrical contact. The cap wafers then have $1 \mu \mathrm{m}$ of thermal oxide grown to serve as an electrical isolation barrier (Fig. 7b). The electrode wafers have $250 \mathrm{~nm}$ of silicon rich nitride deposited on one side to serve as an oxide wet-etch barrier (Fig. 7c). The exposed oxide is removed with a buffered oxide etch (BOE) before bonding to the cap wafers and annealing. The electrodes are defined in the bonded stack with a DRIE halo-etch (Fig. 7d), followed by nitride removal with hot phosphoric acid. The spacer wafers are coated on both sides with $4 \mu \mathrm{m}$ of plasma enhanced chemical vapor deposited (PECVD) silicon oxide to serve as hard masks for a nested etch. On both sides, the PECVD oxide is patterned with reactive ion etching (RIE), followed by DRIE of $450 \mu \mathrm{m}$ to begin defining the aperture (Fig. 7e). The entire wafer is then etched $100 \mu \mathrm{m}$ on each side, followed by an oxide strip (Fig. 7f). The nested etch completes the aperture and defines recesses in the spacer wafer which prevents electrical shorting in the final device. The thin protective oxide on the cap-electrode stacks are removed with BOE. The two stacks and the spacer wafer are then cleaned and fusion bonded, followed by die-sawing to complete the device (Fig. $7 \mathrm{~g}$ ).

Measuring $15 \mathrm{~mm}$ × $4 \mathrm{~mm}$ x $33 \mathrm{~mm}$, the MuSE-QMF contains a $30 \mathrm{~mm}$ quadrupole with an effective electrode diameter of $\sim 1.7 \mathrm{~mm}$, and integrated ion optics (Fig. $8-10$ ).

(a)

(b)
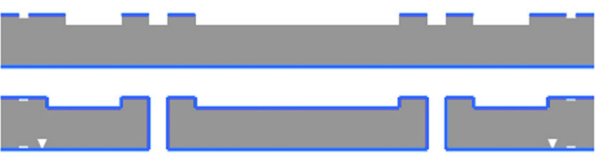

(c)

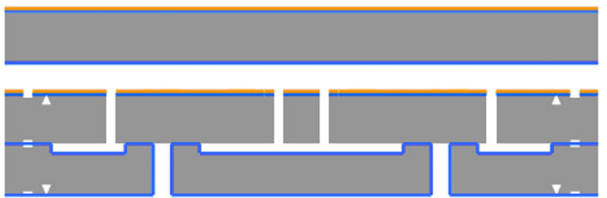

(e)

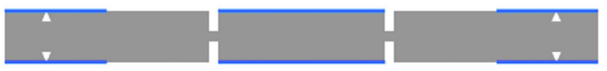

(f)

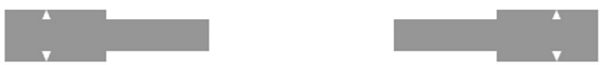

(g)

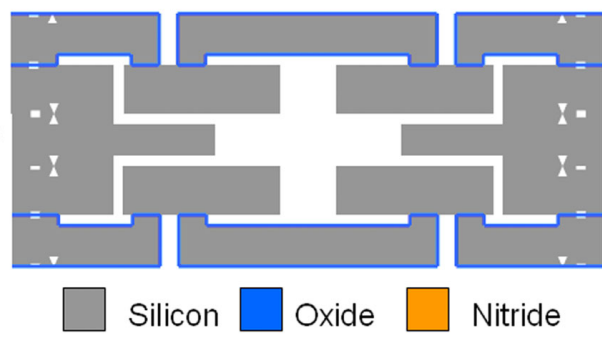

Figure 7: Device process flow - (a) Cap wafer after release trench etch, (b) Completed cap wafer, (c) Electrode wafer with nitride deposition, (d) Bonded cap-electrode stack with patterned electrodes, (e) Spacer wafer with patterned oxide for nested etch, and partial aperture etch, (f) Completed spacer wafer, (g) Final device with two complete cap-electrode stacks bonded to one spacer wafer.

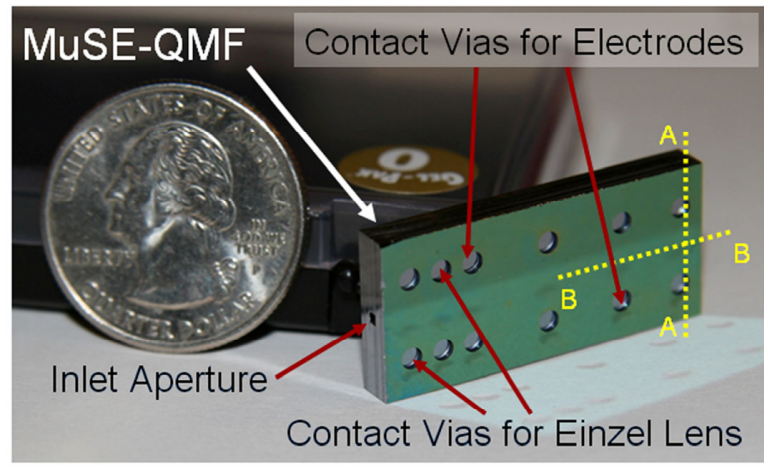

Figure 8: Completed device. The cross section along $A$ is shown in Fig. 9, while the cross section along $B$ is shown in Fig. 10.

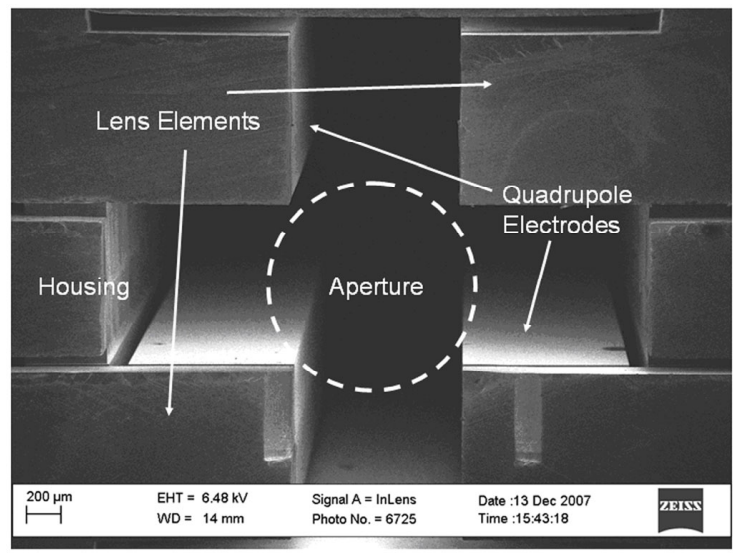

Figure 9: SEM of device interior - cross section across electrodes looking from the exit of the device.

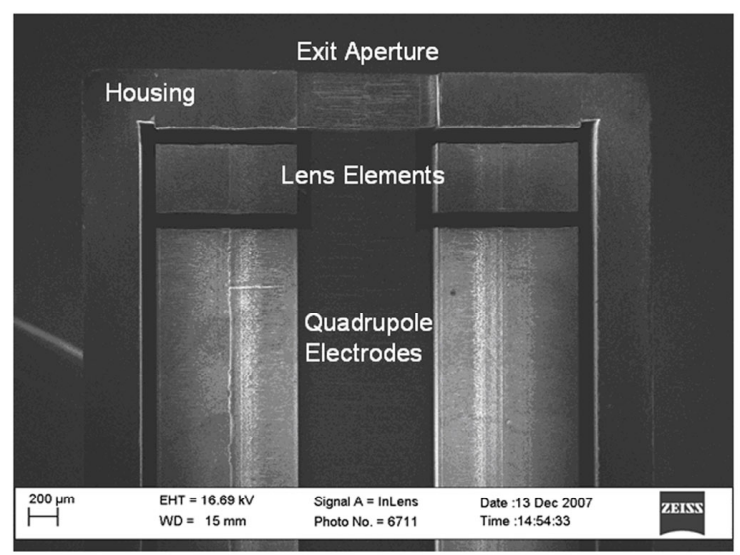

Figure 10: SEM of device interior - cross section along the length of the device at the exit aperture.

\section{EXPERIMENTAL RESULTS}

Testing was conducted at Ardara Technologies (Ardara, PA). The experimental setup comprised of a vacuum chamber that allowed the introduction of air or FC-43 (perfluorotributylamine) into the system at a pressure of $5 \times 10^{-5}$ Torr. A hot filament ionizer was used to generate the ions, and a conversion dynode multiplier was used to detect the signal. A self resonating r.f. supply was set to drive the device at $2 \mathrm{MHz}$ with a maximum voltage of $100 \mathrm{~V}$. A d.c. supply was combined with the r.f. signal through a bias-tee network consisting of $30 \mathrm{M} \Omega$ resistors, and 47 $\mathrm{pF}$ blocking capacitors. Control of the voltage supplies, the ionizer 
current, the detector gain, and the ion optics are managed through a Merlin $\AA$ data acquisition system. The multiplier signal is measured through a preamplifier at a rate of $12.5 \mathrm{kS} / \mathrm{s}$.

A spectrum for FC-43 was obtained in region I to demonstrate the maximum mass range achievable for the MuSE-QMF. Fig. 11 shows a mass range of 250 a.m.u. with prominent peaks at mass 69, 131, and 219. To explore the minimum peak-width obtainable, air was used due to its closely spaced peaks. A spectrum for air was obtained in region II to validate the notion that operation in a higher stability region will enhance resolution, and improve peak shape. Fig. 12 shows a minimum half-height peak-width of 0.7 a.m.u. at mass 28 with clear indication of peaks at mass 32,40 , and 44. The relative peak-intensities of the measured spectra are in good agreement with the NIST library spectra.

This data was obtained without functional integrated ion optics due to structural integrity issues of the lens components. Attempts to drive the device with a voltage greater than $100 \mathrm{~V}$ resulted in electrical breakdown of the insulating oxide. These two limitations diminished the performance of the MuSE-QMF by reducing the maximum mass range achievable, and by exaggerating the peak-shape non-idealities that arise from poor control of the quadrupole inlet and exit conditions.

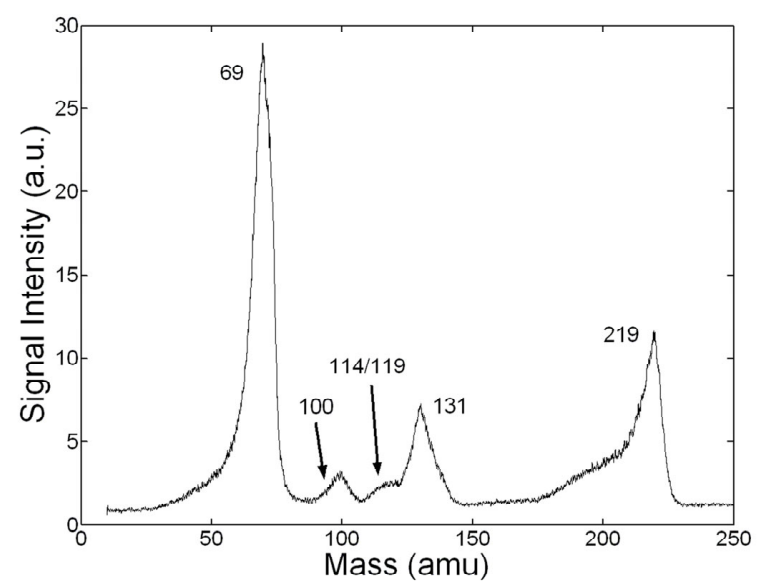

Figure 11: Mass spectrum of FC-43 at $2 \mathrm{MHz}$, Region I. Precursor peaks located on the low mass side of the peaks are apparent.

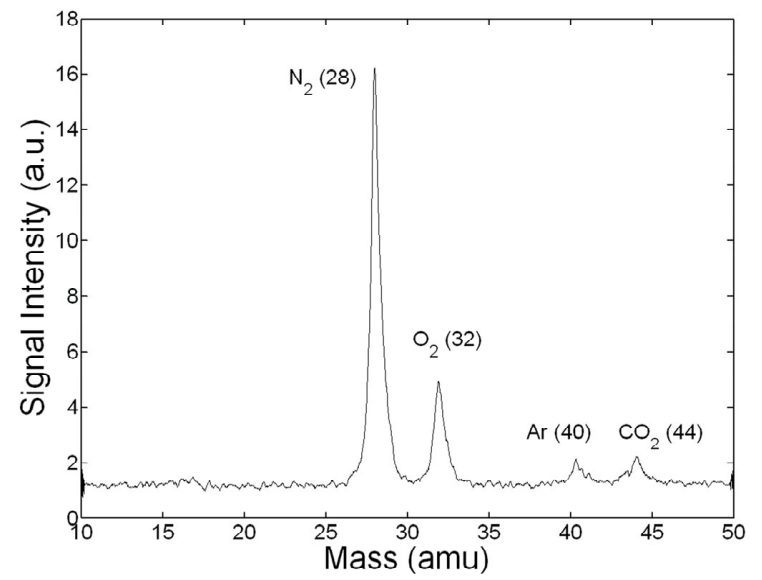

Figure 12: Mass spectrum of air at $2 \mathrm{MHz}$, Region II. Higher resolution and reduced precursor peaks are observed.

\section{CONCLUSIONS}

The ability of a fully batch-fabricated linear quadrupole mass filter with non-conventional square electrodes to perform mass filtering was demonstrated. Potential field calculations with a multipole expansion provided a means of minimizing undesirable higher-order components, thus optimizing the design. Testing of the MuSE-QMF showed increased resolution and improved peakshape when operated in a higher stability region, and the recorded performance is on par with other MEMS-based QMFs. This device design is scalable to smaller dimensions and can readily include integrated pre-filters for enhanced performance.

\section{ACKNOWLEDGEMENTS}

The authors would like to thank Dr. Randy Pedder and the staff at Ardara Technologies (Ardara, PA) for their assistance in the testing of our device. This work was sponsored by DARPA/MTO and the US Army Soldier Systems Center (Natick, MA) through contract \# W911QY-05-1-0002 (DARPA program manager Dennis Polla, and Army program manager Henry Girolamo).

\section{REFERENCES}

[1] S. Taylor, J. R. Gibson, and B. Srigengan, "Miniature mass spectrometry: implications for monitoring of gas discharges", Sensor Review, vol. 23, no. 2, pp. 150 - 154, 2003.

[2] E. R. Badman, and R. G. Cooks, "Miniature mass analyzers", Journal of Mass Spectrometry, vol. 35, pp. 659-671, 2000.

[3] L-Y. Chen, L. F. Velásquez-García, X. Wang, K. Teo, and A. I. Akinwande, "A Micro Ionizer for Portable Mass Spectrometers using Double-gated Isolated Vertically Aligned Carbon Nanofiber Arrays", Technical Digest IEEE International Electron Device Meeting, Washington DC, USA, pp. 843 - 846, December 2007.

[4] Y. Zhu, J. Lee, and A. Seshia, "System-level simulation of a micromachined electrometer using a time-domain variable capacitor circuit model", Journal of Micromechanics and Microengineering, vol. 1, no. 5, pp. 1059 - 1065, 2007.

[5] S. Taylor, R. Tindall, R. R. A. Syms, "Silicon based quadrupole mass spectrometry using microelectromechanical systems", Journal of Vacuum Science and Technology B, vol. 19 , no. 2 , pp. $557-562,2001$.

[6] M. Geear, R. R. A. Syms, S. Wright, and A. S. Holmes, "Monolithic MEMS Quadrupole Mass Spectrometers by Deep Silicon Etching", Journal of Microelectromechanical Systems, vol. 14, no. 5, pp. 1156 - 1166, October 2005.

[7] L. F. Velásquez-García, A. I. Akinwande, "An Out-of-Plane MEMS Quadrupole for a Portable Mass Spectrometer", Technical Digest of the 14th International Conference on Solid-State Sensors, Actuators, and Microsystems, Lyon, France, vol. 2, pp. 2315 - 2320, 2007.

[8] P. Dawson, Quadrupole Mass Spectrometry and Its Applications, AIP, 1997.

[9] L. F. Velásquez-García, K. Cheung, A. I. Akinwande, "An Application of 3D MEMS Packaging: Out-Of-Plane Quadrupole Mass Filters," Journal of Microelectromechanical Systems, submitted for publication.

[10] R. R. A. Syms, T. J. Tate, M. A. Ahmad, S. Taylor, "Design of a microengineered electrostatic quadrupole lens", IEEE Transactions on Electron Devices, vol. 45, no. 11, pp. 2304 2311, November 1998. 